\title{
Acknowledgements to Referees and Staffs for JRACR in 2016
}

\author{
By Chongfu Huang
}

The quality of Journal of Risk Analysis and Crisis Response (JRACR) depends on the qualified and regular collaboration of renowned scientists, who devoted their time to constructively review the submitted articles. I wish to thank the following individuals who acted as referees for the journal in 2016.

It is a great pleasure to thank the staffs serving for JRACR. I appreciate these volunteers who have donated their time without compensation to promote the journal.

\section{Referees of JRACR:}

$\begin{array}{llll}\text { Christopher C. Allan } & \text { Jinqiu Hu } & \text { Tiezhong Liu } & \text { Geetam Tiwari } \\ \text { Agnes Allansdottir } & \text { Carel TJ Hulshof } & \text { Xilin Liu } & \text { Ruipeng Tong } \\ \text { Arjen Boin } & \text { Chengjun Ji } & \text { Yun Luo } & \text { Donald A. Wilhite } \\ \text { An Chen } & \text { Tae-Woong Kim } & \text { Jianming Mo } & \text { Chaowu Xie } \\ \text { Gianpaolo Cimellaro } & \text { Emérite André Laurent } & \text { Bin Pan } & \text { Xinliang Xu } \\ \text { Lei Deng } & \text { Supeng Leng } & \text { Lei Pang } & \text { Xuanhua Xu } \\ \text { Min Ding } & \text { Husheng Li } & \text { A. Pearson } & \text { Fei You } \\ \text { Christoph Doktor } & \text { Jingyi Li } & \text { Andy Pearson } & \text { Zhuliang Yu } \\ \text { Edna Einsiedel } & \text { Qiang Li } & \text { Salvador Casadesús Pursals } & \text { Hongmei Zhang } \\ \text { Alexander Fekete } & \text { Shuangshuang Li } & \text { Huanguang Qiu } & \text { Junye Zhao } \\ \text { Zhaoyong Guan } & \text { Xu Li } & \text { Manuel D. Rossetti } & \\ \text { Zhenhua Guo } & \text { Kai Liu } & \text { Yanrui Shang } & \end{array}$

\section{Staffs of JRACR:}

\section{Editorial Board Office}

Mu Zhang

Hongmei Zhang

Wen Han

Ziyan Wang

Xiaobo Lu

\author{
Editorial Department \\ Junxiang Zhang \\ Fei Wen \\ Hongbing Zhu \\ Jingjing Cheng
}

\author{
Reviewer Center \\ Xilei Pang \\ Ye Xue \\ Fuping Yang
}

Chongfu Huang, Professor, Ph.D. Academy of Disaster Reduction and Emergency Management

Beijing Normal University No.19 Xinjiekouwai Street Beijing 100875, China 